\title{
Discrete Waveform Relaxation Method for Linear Fractional Delay Differential-Algebraic Equations
}

\author{
Hongliang Liu, Yayun Fu, and Bailing Li \\ Hunan Key Laboratory for Computation and Simulation in Science and Engineering, Xiangtan University, Xiangtan, \\ Hunan 411105, China \\ Correspondence should be addressed to Hongliang Liu; lhl@xtu.edu.cn
}

Received 3 October 2016; Accepted 19 January 2017; Published 15 June 2017

Academic Editor: Silvia Romanelli

Copyright (c) 2017 Hongliang Liu et al. This is an open access article distributed under the Creative Commons Attribution License, which permits unrestricted use, distribution, and reproduction in any medium, provided the original work is properly cited.

Fractional order delay differential-algebraic equations have the characteristics of time lag and memory and constraint limit. These yield some difficulties in the theoretical analysis and numerical computation. In this paper, we are devoted to solving them by the waveform relaxation method. The corresponding convergence results are obtained, and some numerical examples show the efficiency of the method.

\section{Introduction}

Fractional delay differential-algebraic equations are composed of fractional delay differential equations and algebraic equations, and they are more accurate in describing some scientific and engineering problems with memory function and algebraic restrictions. This kind of mathematical model is widely applied in many fields of electromagnetism, electrical systems, and biological materials. Nowadays, for the fractional differential equations, the main works focus on obtaining the analytic solution, approximately analytical solution, and numerical solution. For example, the Laplace transform method [1, 2], Fourier transform method [3, 4], and power method [5] are used to obtain the analytic solution. Furthermore, approximate analytical methods have been developed, such as variational iteration method [6, 7], Adomian decomposition method [8], homotopy analysis method [9], and multiple time scale method [10]. As for the studies of numerical methods, scholars discuss the linear multistep method [11], predictor corrector method [12, 13], Adomian method [14-16], spectrum method, and the finite element method $[17,18]$ for solving the fractional differential equations.

The waveform relaxation (WR) method is proposed by Learsmee and so forth [19]. The main properties of the method are flexibility and good parallelism, which is used to solve differential equations [20,21], fractional differential equations [22, 23], delay differential equations $[24,25]$, and differential-algebraic equations [26-28]. The waveform relaxation method based on multisplitting is proposed to improve parallelism $[29,30]$. Furthermore, acceleration techniques are used to increase the speed of iteration, such as Krylov subspace acceleration, strange-type preconditioners, and windowing for the WR method [20,21, 31, 32].

The fractional differential-algebraic equations have received much attention; nevertheless, the numerical methods in this field are still young; a few studies have been considered on the convergence of the numerical methods, such as variational iteration method, Adomian decomposition method, and fractional differential transform method [33,34]. Ding and Jiang applied the WR method to solve fractional differential-algebraic equations and obtain the convergence results [35]. But it is difficult to verify convergence conditions. The variational iteration method for the fractional delay integrodifferential-algebraic equations has been studied in [36], but it is not suitable for long-time numerical calculation. In order to overcome this limitation, according to the characteristics of the problems, the discrete WR method is employed to solve the linear fractional delay differential-algebraic equations by constructing the efficient iterative method and obtain the convergence results which is much easier to achieve. 


\section{Convergence}

Consider the Caputo fractional delay differential-algebraic system

$$
\begin{aligned}
& M_{a}^{C} D_{0}^{\alpha} x(t)+A x(t)+B y(t)+C x(t-\tau)+D y(t-\tau) \\
& \quad=U(t), \quad t \in[0, T] \\
& E x(t)+N y(t)+F x(t-\tau)+G y(t-\tau)=V(t),
\end{aligned}
$$$$
t \in[0, T],
$$$$
x(0)=y(0)=0, \quad t \in[0, T],
$$

where $D_{*}^{\alpha} x(t)$ denotes the Caputo derivative of order $\alpha, n_{1}-$ $1<\alpha \leq n_{1}, n_{1} \in N, \varphi(t), \psi(t)$ are smooth vector functions, and $\varphi(0)=0, \psi(0)=0, M \in \mathfrak{R}^{n \times n}, N \in \mathfrak{R}^{d \times d}$ are nonsingular matrix, $A, C \in \mathfrak{R}^{n \times n}, B, D \in \mathfrak{R}^{n \times d}, E, F \in \mathfrak{R}^{d \times n}$, $G \in \mathfrak{R}^{d \times d}$, and $x(t) \in C^{n}[0, T], y(t) \in \mathfrak{R}^{d}, n+d=s$, $U(t) \in \mathfrak{R}^{n}, V(t) \in \mathfrak{R}^{d}$ are input function.

Applying the WR method for the coefficient matrix of (1), we get the iteration form

$$
\begin{aligned}
& M_{1}{ }_{a}^{C} D_{0}^{\alpha} x^{(k+1)}(t)+A_{1} x^{(k+1)}(t)+B_{1} y^{(k+1)}(t) \\
& \quad+C_{1} x^{(k+1)}(t-\tau)+D_{1} y^{(k+1)}(t-\tau) \\
& \quad=M_{2}{ }_{a}^{C} D_{0}^{\alpha} x^{(k)}(t)+A_{2} x^{(k)}(t)+B_{2} y^{(k)}(t) \\
& \quad+C_{2} x^{(k)}(t-\tau)+D_{2} y^{(k)}(t-\tau)+U(t), \\
& E_{1} x^{(k+1)}(t)+N_{1} y^{(k+1)}(t)+F_{1} x^{(k+1)}(t-\tau) \\
& \quad+G_{1} y^{(k+1)}(t-\tau)=E_{2} x^{(k)}(t)+N_{2} y^{(k)}(t) \\
& \quad+F_{2} x^{(k)}(t-\tau)+G_{2} y^{(k)}(t-\tau)+V(t), \\
& x^{(k+1)}(t)=x^{(k)}(t)=\cdots=x^{(0)}(t)=\varphi(t), \quad t \in[-\tau, 0], \\
& y^{(k+1)}(t)=y^{(k)}(t)=\cdots=y^{(0)}(t)=\psi(t), \\
& \quad k=0,1, \ldots,
\end{aligned}
$$

where $A=A_{1}-A_{2}, B=B_{1}-B_{2}, C=C_{1}-C_{2}, D=D_{1}-D_{2}$, $E=E_{1}-E_{2}, F=F_{1}-F_{2}, G=G_{1}-G_{2}, M=M_{1}-M_{2}$, $N=N_{1}-N_{2}$, and $M_{1}, N_{1}$ are nonsingular matrix. We assume that the step is $h, \tau=m h, m \geq 1, t_{p-j}=t_{p}-j h, p \geq 1, x_{p-j}^{(k)}$ is approximating value of $x^{(k)}\left(t_{p-j}\right)$, and $y_{p-j}^{(k)}$ is approximating value of $y^{(k)}\left(t_{p-j}\right), j=0,1, \ldots, p$.

Based on the fractional Caputo derivative of GrünwaldLetnikov discrete format

$$
{ }_{a}^{C} D_{0}^{\alpha} x(t) \doteq \frac{1}{h^{\alpha}} \sum_{j=0}^{p} \omega_{j} x_{p-j},
$$

where $\omega_{j}=(-1)^{j}\left(\begin{array}{l}\alpha \\ j\end{array}\right)=(-1)^{j}(\Gamma(j-\alpha) / \Gamma(-\alpha) \Gamma(j+1))$ and $\left(\begin{array}{l}\alpha \\ 0\end{array}\right)=\Gamma(-\alpha) / \Gamma(-\alpha) \Gamma(1)=1$, we obtain the iterative format

$$
\begin{gathered}
\left(M_{1}+h^{\alpha} A_{1}\right) x_{p}^{(k+1)}+\sum_{j=1}^{p} \omega_{j} M_{1} x_{p-j}^{(k+1)}+h^{\alpha} \bar{v}_{n} \\
=\left(M_{2}+h^{\alpha} A_{2}\right) x_{p}^{(k)}+\sum_{j=1}^{p} \omega_{j} M_{2} x_{p-j}^{(k)}+h^{\alpha} \bar{u}_{n}, \\
E_{1} x_{p}^{(k+1)}+N_{1} y_{p}^{(k+1)}+F_{1} x_{p-m}^{(k+1)}+G_{1} y_{p-m}^{(k+1)} \\
=E_{2} x_{p}^{(k)}+N_{2} y_{p}^{(k)}+F_{2} x_{p-m}^{(k)}+G_{2} y_{p-m}^{(k)}+V\left(t_{p}\right), \\
x^{(k+1)}(t)=x^{(k)}(t)=\cdots=x^{(0)}(t)=\varphi(t), \\
y^{(k+1)}(t)=y^{(k)}(t)=\cdots=y^{(0)}(t)=\psi(t), \\
p=1,2, \ldots, N, \quad k=0,1, \ldots,
\end{gathered}
$$

where

$$
\begin{aligned}
& \bar{v}_{n}=B_{1} y_{p}^{(k+1)}+C_{1} x_{p-m}^{(k+1)}+D_{1} y_{p-m}^{(k+1)}, \\
& \bar{u}_{n}=B_{2} y_{p}^{(k)}+C_{2} x_{p-m}^{(k)}+D_{2} y_{p-m}^{(k)}+U\left(t_{p}\right),
\end{aligned}
$$

where $N$ is the number of the time steps and $N h=T$, the initial value $x_{0}^{(0)}=0$. Introduce

$$
\begin{aligned}
& X^{(k)}=\left[\left(X_{1}^{(k)}\right)^{T},\left(X_{2}^{(k)}\right)^{T}, \ldots,\left(X_{p}^{(k)}\right)^{T}\right]^{T}, \\
& Y^{(k)}=\left[\left(Y_{1}^{(k)}\right)^{T},\left(Y_{2}^{(k)}\right)^{T}, \ldots,\left(Y_{p}^{(k)}\right)^{T}\right]^{T} .
\end{aligned}
$$

Next, we will discuss two cases.

First Case. When $p<m$, (4) can be written as

$$
\begin{aligned}
& {\left[\begin{array}{cc}
H_{1} & h^{\alpha}\left(I \otimes B_{1}\right) \\
I \otimes E_{1} & I \otimes N_{1}
\end{array}\right]\left[\begin{array}{c}
X^{(k+1)} \\
Y^{(k+1)}
\end{array}\right]} \\
& \quad=\left[\begin{array}{cc}
H_{2} & h^{\alpha}\left(I \otimes B_{2}\right) \\
I \otimes E_{2} & I \otimes N_{2}
\end{array}\right]\left[\begin{array}{c}
X^{(k)} \\
Y^{(k)}
\end{array}\right]+\left[\begin{array}{c}
\widehat{Z} \\
\widehat{Q}
\end{array}\right],
\end{aligned}
$$

where $H_{i}=M_{\alpha} \otimes M_{i}+h^{\alpha}\left(I \otimes A_{i}\right), \quad i=1,2$, and

$$
M_{\alpha}=\left[\begin{array}{cccc}
w_{0} & & & \\
w_{1} & w_{0} & & \\
\vdots & \ddots & \ddots & \\
w_{p-1} & \ddots & w_{1} & w_{0}
\end{array}\right] \text {; }
$$


introduce $\widehat{Z}=\left[\widehat{z}_{1}^{T}, \widehat{z}_{2}^{T}, \ldots, \widehat{z}_{i}^{T}, \ldots, \widehat{z}_{p}^{T}\right]^{T}, \widehat{Q}=\left[\widehat{q}_{1}^{T}, \widehat{q}_{2}^{T}, \ldots\right.$, $\left.\hat{q}_{i}^{T}, \ldots, \hat{q}_{p}^{T}\right]^{T}$, where

$$
\begin{aligned}
\widehat{z}_{i}=h^{\alpha}\left(U\left(t_{i}\right)-C \varphi\left(t_{i-m}\right)-D \psi\left(t_{i-m}\right)\right), & \\
& i=1,2, \ldots, p, \\
\widehat{q}_{i}=V\left(t_{i}\right)-F \varphi\left(t_{i-m}\right)-G \psi\left(t_{i-m}\right), & i=1,2, \ldots, p .
\end{aligned}
$$
$1, \ldots$

Let $\delta_{1}^{(k+1)}=\left[\left(X^{(k+1)}-X^{(k)}\right)^{T},\left(Y^{(k+1)}-Y^{(k)}\right)^{T}\right]^{T}, k=0$,

From (7), we have

$$
\begin{aligned}
\delta_{1}^{(k+1)}= & {\left[\begin{array}{cc}
H_{1} & h^{\alpha}\left(I \otimes B_{1}\right) \\
I \otimes E_{1} & I \otimes N_{1}
\end{array}\right]^{-1} } \\
& \cdot\left[\begin{array}{cc}
H_{2} & h^{\alpha}\left(I \otimes B_{2}\right) \\
I \otimes E_{2} & I \otimes N_{2}
\end{array}\right] \delta_{1}^{(k)} .
\end{aligned}
$$

Let the iterative matrix be $R_{1}$ in (10); the convergence condition of the iterative process $(4)$ is $\rho\left(R_{1}\right)<1$. Moreover, we have the following theorem.

Theorem 1. For the small step $h$ and $p<m$, if

$$
\begin{gathered}
\rho\left(M_{1}^{-1} M_{2}\right)<1, \\
\rho\left(N_{1}^{-1} N_{2}\right)<1,
\end{gathered}
$$

then the discrete waveform relaxation iteration method (4) is convergent.

Proof. The iteration process (4) convergence is decided by $\bar{R}_{1}$. Firstly, the left coefficient matrix of (7) can be written as

$$
\begin{aligned}
& {\left[\begin{array}{cc}
H_{1} & h^{\alpha}\left(I \otimes B_{1}\right) \\
I \otimes E_{1} & I \otimes N_{1}
\end{array}\right]} \\
& \quad=\left[\begin{array}{cc}
M_{\alpha} \otimes M_{1} & 0 \\
I \otimes E_{1} & I \otimes N_{1}
\end{array}\right]+h^{\alpha}\left[\begin{array}{cc}
I \otimes A_{1} & I \otimes B_{1} \\
0 & 0
\end{array}\right],
\end{aligned}
$$

because of the following equation:

$$
\begin{aligned}
& {\left[\begin{array}{cc}
M_{\alpha} \otimes M_{1} & 0 \\
I \otimes E_{1} & I \otimes N_{1}
\end{array}\right]^{-1}} \\
& \quad=\left[\begin{array}{cc}
\left(M_{\alpha} \otimes M_{1}\right)^{-1} & 0 \\
W_{1} & \left(I \otimes N_{1}\right)^{-1}
\end{array}\right],
\end{aligned}
$$

where $W_{1}=-\left(I \otimes N_{1}\right)^{-1}\left(I \otimes E_{1}\right)\left(M_{\alpha} \otimes M_{1}\right)^{-1}$; then,

$$
\begin{aligned}
& {\left[\begin{array}{cc}
H_{1} & h^{\alpha}\left(I \otimes B_{1}\right) \\
I \otimes E_{1} & I \otimes N_{1}
\end{array}\right]^{-1}} \\
& \quad=\left[\begin{array}{cc}
\left(M_{\alpha} \otimes M_{1}\right)^{-1} & 0 \\
W_{1} & \left(I \otimes N_{1}\right)^{-1}
\end{array}\right]^{-1}+O\left(h^{\alpha}\right),
\end{aligned}
$$

and we can get

$R_{1}$

$$
\begin{aligned}
= & \left(\left[\begin{array}{cc}
\left(M_{\alpha} \otimes M_{1}\right)^{-1} & 0 \\
W_{1} & \left(I \otimes N_{1}\right)^{-1}
\end{array}\right]+O\left(h^{\alpha}\right)\right) \\
& \cdot\left(\left[\begin{array}{cc}
M_{\alpha} \otimes M_{2} & 0 \\
I \otimes E_{2} & I \otimes N_{2}
\end{array}\right]+h^{\alpha}\left[\begin{array}{cc}
I \otimes A_{2} & I \otimes B_{2} \\
0 & 0
\end{array}\right]\right) \\
= & {\left[\begin{array}{cc}
\left(M_{\alpha} \otimes M_{1}^{-1}\right)\left(M_{\alpha} \otimes M_{2}\right) & 0 \\
\widetilde{W}_{1} & \left(I \otimes N_{1}\right)^{-1}\left(I \otimes N_{2}\right)
\end{array}\right] } \\
& +O\left(h^{\alpha}\right),
\end{aligned}
$$

where $\widetilde{W}_{1}=W_{1}\left(M_{\alpha} \otimes M_{2}\right)+\left(I \otimes N_{1}\right)^{-1}\left(I \otimes N_{2}\right)$; we have

$$
\rho\left(\left(M_{\alpha} \otimes M_{1}\right)^{-1}\left(M_{\alpha} \otimes M_{2}\right)\right)=\rho\left(M_{1}^{-1} M_{2}\right) .
$$

Based on the matrix spectral radius definition of $R_{1}$ expression in (15), if

$$
\begin{gathered}
\rho\left(M_{1}^{-1} M_{2}\right)<1, \\
\rho\left(N_{1}^{-1} N_{2}\right)<1,
\end{gathered}
$$

and $h$ is small enough, we have $\rho\left(R_{1}\right)<1$.

Therefore, when $p<m$, the convergence is proved.

Second Case. When $p>m$, we assume $p=m+c$; then, system (4) can be written as

$$
\left[\begin{array}{ll}
\bar{H}_{1} & \bar{N}_{1} \\
\bar{K}_{1} & \bar{L}_{1}
\end{array}\right]\left[\begin{array}{l}
X^{(k+1)} \\
Y^{(k+1)}
\end{array}\right]=\left[\begin{array}{ll}
\bar{H}_{2} & \bar{N}_{2} \\
\bar{K}_{2} & \bar{L}_{2}
\end{array}\right]\left[\begin{array}{l}
X^{(k)} \\
Y^{(k)}
\end{array}\right]+\left[\begin{array}{l}
\widehat{S} \\
\widehat{T}
\end{array}\right],
$$

where

$$
\begin{aligned}
& \bar{H}_{i}=M_{\alpha} \otimes M_{i}+h^{\alpha}\left(I \otimes A_{i}+\bar{P}_{\xi} \otimes C_{i}\right), \quad i=1,2, \\
& \bar{N}_{i}=h^{\alpha}\left(I \otimes B_{i}+\bar{P}_{\xi} \otimes D_{i}\right), \quad i=1,2, \\
& \bar{K}_{i}=I \otimes E_{i}+\bar{P}_{\xi} \otimes F_{i}, \quad i=1,2, \\
& \bar{L}_{i}=I \otimes N_{i}+\bar{P}_{\xi} \otimes G_{i}, \quad i=1,2,
\end{aligned}
$$




$$
\bar{P}_{\xi}=\left(\begin{array}{cccccc}
0 & & & & & \\
0 & 0 & & & & \\
\vdots & \ddots & \ddots & & & \\
1 & & 0 & 0 & & \\
\vdots & \ddots & & \ddots & \ddots & \\
0 & \cdots & 1 & \cdots & 0 & 0
\end{array}\right)
$$

where

$$
\begin{aligned}
\bar{P}_{\xi} & =\left(\xi_{i j}\right) \\
& = \begin{cases}1, & i=m, m+1, \ldots, N, j=i-m+1, \\
0, & \text { others; }\end{cases}
\end{aligned}
$$

introduce $\widehat{S}=\left[\widehat{s}_{1}^{T}, \widehat{s}_{2}^{T}, \ldots, \widehat{s}_{i}^{T}, \ldots, \widehat{s}_{p}^{T}\right]^{T}, \widehat{T}=\left[\widehat{t}_{1}^{T}, \hat{t}_{2}^{T}, \ldots\right.$, $\left.\widehat{t}_{i}^{T}, \ldots, \hat{t}_{p}^{T}\right]^{T}$, where $\widehat{s}_{i}, \widehat{t}_{i}$ are

$$
\begin{gathered}
\widehat{s}_{i} \\
= \begin{cases}h^{\alpha}\left(U\left(t_{i}\right)-C \varphi\left(t_{i-m}\right)-D \psi\left(t_{i-m}\right)\right), & i=1,2, \ldots, m-1, \\
h^{\alpha} U\left(t_{i}\right), & i=m, m+1, \ldots, p,\end{cases} \\
\widehat{t}_{i}= \begin{cases}V\left(t_{i}\right)-F \varphi\left(t_{i-m}\right)-G \psi\left(t_{i-m}\right), & i=0, \ldots, m-1, \\
V\left(t_{i}\right), & i=m, m+1, \ldots, p .\end{cases}
\end{gathered}
$$

Let $\widetilde{\delta}_{1}^{(k+1)}=\left[\left(X^{(k+1)}-X^{(k)}\right)^{T},\left(Y^{(k+1)}-Y^{(k)}\right)^{T}\right]^{T}, k=0$, $1, \ldots$, and from (18) we have

$$
\widetilde{\delta}_{1}^{(k+1)}=\left[\begin{array}{ll}
\bar{H}_{1} & \bar{N}_{1} \\
\bar{K}_{1} & \bar{L}_{1}
\end{array}\right]^{-1} \cdot\left[\begin{array}{ll}
\bar{H}_{2} & \bar{N}_{2} \\
\bar{K}_{2} & \bar{L}_{2}
\end{array}\right] \widetilde{\delta}_{1}^{(k)} .
$$

Let the iterative matrix be $R_{2}$ in (22); then, the convergence condition of the iterative process $(22)$ is $\rho\left(R_{2}\right)<1$; we have the following theorem.

Theorem 2. For the small step $h$, if it satisfies

$$
\begin{aligned}
& \rho\left(M_{1}^{-1} M_{2}\right)<1, \\
& \rho\left(N_{1}^{-1} N_{2}\right)<1,
\end{aligned}
$$

then the discrete waveform relaxation iteration method (18) is convergent.
Proof. The iteration process (18) convergence is decided by $R_{2}$. Firstly, the left coefficient matrix of (18) can be written as

$$
\begin{aligned}
& {\left[\begin{array}{cc}
\bar{H}_{1} & \bar{N}_{1} \\
\bar{K}_{1} & \bar{L}_{1}
\end{array}\right]} \\
& \quad=\left[\begin{array}{cc}
M_{\alpha} \otimes M_{1} & 0 \\
\bar{K}_{1} & \bar{L}_{1}
\end{array}\right] \\
& \quad+h^{\alpha}\left[\begin{array}{cc}
I \otimes A_{1}+\bar{P}_{\xi} \otimes C_{1} & I \otimes B_{1}+\bar{P}_{\xi} \otimes D_{1} \\
0 & 0
\end{array}\right],
\end{aligned}
$$

and because

$$
\begin{aligned}
& \bar{L}_{1}^{-1} \\
& =\left[\begin{array}{cccccc}
N_{1}^{-1} & & & & & \\
0 & N_{1}^{-1} & & & & \\
\vdots & \ddots & \ddots & & & \\
-N_{1}^{-1} G_{1} N_{1}^{-1} & & 0 & N_{1}^{-1} & \\
& \ddots & & \ddots & \ddots & \\
0 & & -N_{1}^{-1} G_{1} N_{1}^{-1} & \cdots & 0 & N_{1}^{-1}
\end{array}\right] \\
& =\left(I \otimes N_{1}\right)^{-1}+\left(\begin{array}{cc}
0 & 0 \\
\widetilde{L}_{1} & 0
\end{array}\right), \\
&
\end{aligned}
$$

where $\widetilde{L}_{1}=-\bar{P}_{\xi} \otimes\left(-N_{1}^{-1} G_{1} N_{1}^{-1}\right)$, hence

$$
\left[\begin{array}{cc}
M_{\alpha} \otimes M_{1} & 0 \\
\bar{K}_{1} & \bar{L}_{1}
\end{array}\right]^{-1}=\left[\begin{array}{cc}
\left(M_{\alpha} \otimes M_{1}\right)^{-1} & 0 \\
W_{2} & \bar{L}_{1}^{-1}
\end{array}\right],
$$

where $W_{2}=-L_{1}^{-1}\left(I \otimes E_{1}+\bar{P}_{\xi} \otimes F_{1}\right)\left(M_{\alpha} \otimes M_{1}\right)^{-1}$; then,

$$
\begin{aligned}
{\left[\begin{array}{cc}
\bar{H}_{1} & \bar{N}_{1} \\
\bar{K}_{1} & \bar{L}_{1}
\end{array}\right]^{-1}=} & {\left[\begin{array}{cc}
\left(M_{\alpha} \otimes M_{1}\right)^{-1} & 0 \\
\widehat{W}_{2} & \left(I \otimes N_{1}\right)^{-1}
\end{array}\right] } \\
& +O\left(h^{\alpha}\right),
\end{aligned}
$$

where $\bar{W}_{2}=W_{2}+\widetilde{L}_{1}$; we have

$$
\begin{aligned}
R_{2} & =\left(\left[\begin{array}{cc}
\left(M_{\alpha} \otimes M_{1}\right)^{-1} & 0 \\
W_{2} & \bar{L}_{1}^{-1}
\end{array}\right]+O\left(h^{\alpha}\right)\right) \\
& \cdot\left(\left[\begin{array}{cc}
M_{\alpha} \otimes M_{2} & 0 \\
\bar{K}_{2} & \bar{L}_{2}
\end{array}\right]\right. \\
& \left.+h^{\alpha}\left[\begin{array}{cc}
I \otimes A_{2}+\bar{P}_{\xi} \otimes C_{2} & I \otimes B_{2}+\bar{P}_{\xi} \otimes D_{2} \\
0 & 0
\end{array}\right]\right) \\
& =\left[\begin{array}{cc}
\left(M_{\alpha} \otimes M_{1}^{-1}\right)\left(M_{\alpha} \otimes M_{2}\right) & 0 \\
\widetilde{W}_{2} & \bar{L}_{1}^{-1} \bar{L}_{2}
\end{array}\right]+O\left(h^{\alpha}\right),
\end{aligned}
$$

where $\widetilde{W}_{2}=\bar{W}_{2}\left(M_{\alpha} \otimes M_{2}\right)+\bar{L}_{1}^{-1} \bar{L}_{2}$, and because 


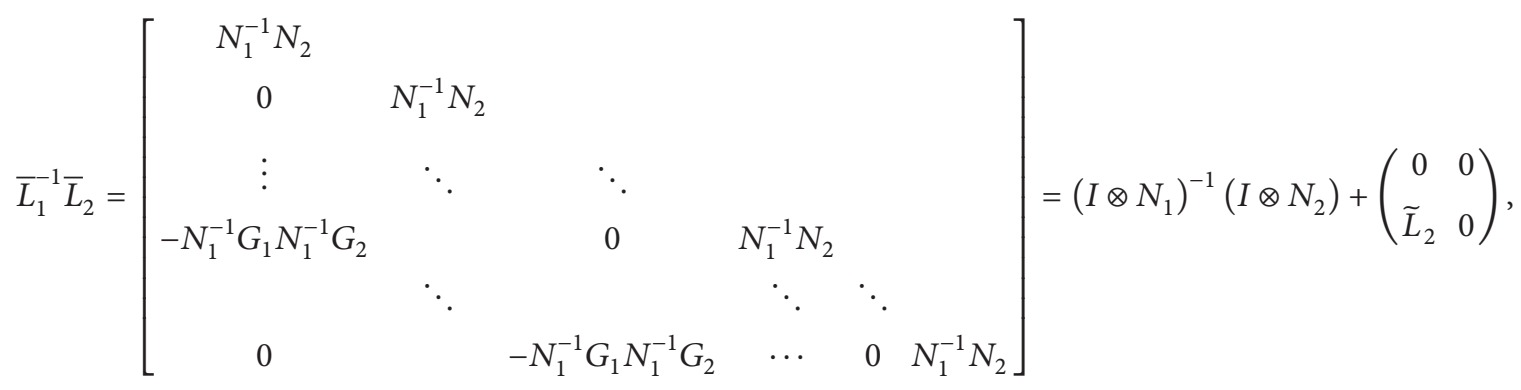

where $\widetilde{L}_{2}=-\bar{P}_{\xi} \otimes\left(N_{1}^{-1} G_{1} N_{2}^{-1} G_{2}\right)$, therefore, we have

$$
\rho\left(\bar{L}_{1}^{-1} \bar{L}_{2}\right)=\rho\left(N_{1}^{-1} N_{2}\right)
$$

from (8), we have

$$
\rho\left(\left(M_{\alpha} \otimes M_{1}\right)^{-1}\left(M_{\alpha} \otimes M_{2}\right)\right)=\rho\left(M_{1}^{-1} M_{2}\right) .
$$

Based on the matrix spectral radius definition and combining $R_{2}$ expression of (28) and if

$$
\begin{gathered}
\rho\left(M_{1}^{-1} M_{2}\right)<1, \\
\rho\left(N_{1}^{-1} N_{2}\right)<1,
\end{gathered}
$$

and $h$ is enough small, we have $\rho\left(R_{2}\right)<1$.

Therefore, the convergence is proved.

\section{Illustrative Examples}

In this section, some illustrative examples are given to show the efficiency of the WR method for solving the linear fractional delay differential-algebraic equations.

Example 1. Consider the initial value problem of the linear fractional delay differential-algebraic equations

$$
\begin{aligned}
& {\left[\begin{array}{ccc}
-5 & 1 & 0 \\
1 & 5 & 0 \\
0 & 0 & 0
\end{array}\right]{ }_{a}^{C} D_{0}^{\alpha} x(t)+\left[\begin{array}{ccc}
-0.1 & 0 & 0 \\
0 & 0.3 & 0.5 \\
0.001 & 0 & -0.5
\end{array}\right] x(t)} \\
& +\left[\begin{array}{ccc}
0.12 & 0.2 & 0 \\
0 & 0.1 & -0.5 \\
0 & 0 & 0.8
\end{array}\right] x(t-0.5)=\left[\begin{array}{c}
\sin (t) \\
\sin (t) \\
0
\end{array}\right], \\
& \left.x(t)=(0,0,0)^{T}, \quad t \in[-0.5,0], T\right],
\end{aligned}
$$

where $\alpha=0.5, x(t)=\left(x_{1}(t), x_{2}(t), x_{3}(t)\right)^{T}$; introduce $\widetilde{x}(t)=$ $\left(x_{1}(t), x_{2}(t)\right)^{T}, y(t)=x_{3}(t)$; system (33) can be written as

$$
\begin{aligned}
& M_{a}^{C} D_{0}^{\alpha} \tilde{x}(t)+A \tilde{x}(t)+B y(t)+C \tilde{x}(t-0.5) \\
+D y(t-0.5)=U(t), \quad t \in[0, T], & \\
E \tilde{x}(t)+N y(t)+F \tilde{x}(t-0.5)+G y(t-0.5) & =V(t), \\
t & \in[0, T],
\end{aligned}
$$

$$
\begin{aligned}
& \tilde{x}(t)=(0,0)^{T}, \\
& y(t)=0,
\end{aligned}
$$

$$
t \in[-0.5,0],
$$

and we have

$$
\begin{aligned}
& M=\left[\begin{array}{cc}
-5 & 1 \\
1 & 5
\end{array}\right], \\
& A=\left[\begin{array}{cc}
-0.1 & 0 \\
0 & 0.3
\end{array}\right] \text {, } \\
& B=\left[\begin{array}{c}
0 \\
0.5
\end{array}\right] \text {, } \\
& C=\left[\begin{array}{cc}
0.12 & 0.2 \\
0 & 0.1
\end{array}\right], \\
& D=\left[\begin{array}{c}
0 \\
-0.5
\end{array}\right] \text {, } \\
& E=\left[\begin{array}{ll}
0.001 & 0
\end{array}\right], \\
& N=-0.5 \text {, } \\
& F=\left[\begin{array}{ll}
0 & 0
\end{array}\right], \\
& G=0.8, \\
& U(t)=\left[\begin{array}{ll}
\sin (t) & \sin (t)
\end{array}\right]^{T}, \\
& V(t)=0 .
\end{aligned}
$$


TABLE 1: The error of numerical solutions.

\begin{tabular}{lcccc}
\hline$T$ & $T=1$ & $T=2$ & $T=3$ & $T=4$ \\
\hline$\tilde{x}_{1}(t)$ & $3.0981 e-003$ & $1.1308 e-003$ & $2.3020 e-003$ & $3.8279 e-003$ \\
$\tilde{x}_{2}(t)$ & $4.1365 e-003$ & $1.1548 e-003$ & $3.5419 e-003$ & $5.4337 e-003$ \\
$y_{1}(t)$ & $1.9550 e-005$ & $3.4345 e-004$ & $1.6464 e-003$ & $5.455 e-003$ \\
\hline
\end{tabular}

Splitting the coefficient matrix, we can construct the $J a c o b i$ waveform relaxation method and deduce

$$
\begin{aligned}
& M_{1}=\left[\begin{array}{ll}
-5 & 0 \\
0 & 5
\end{array}\right], \\
& M_{2}=\left[\begin{array}{ll}
0 & 1 \\
-1 & 0
\end{array}\right], \\
& A_{1}=\left[\begin{array}{ll}
-0.1 & 0 \\
0 & -0.3
\end{array}\right], \\
& A_{2}=\left[\begin{array}{ll}
0 & 0 \\
0 & 0
\end{array}\right], \\
& N_{1}=-\frac{0.5}{1.1}, \\
& F_{1}=\left[\begin{array}{ll}
0 \\
E_{1}
\end{array}\right], \\
& D_{2}=\left[\begin{array}{ll}
0.05 \\
0.5
\end{array}\right], \\
& B_{2}=\left[\begin{array}{ll}
0 & 0.1 \\
B_{1} & =\left[\begin{array}{ll}
0 \\
0
\end{array}\right], \\
0
\end{array}\right],
\end{aligned}
$$

TABLE 2: Between the iteration allowable error and the number of iterations.

\begin{tabular}{lccc}
\hline$T$ & $1.0 \times 10^{-4}$ & $1.0 \times 10^{-6}$ & $1.0 \times 10^{-8}$ \\
\hline$T=1$ & 5 & 7 & 10 \\
$T=2$ & 4 & 7 & 10 \\
$T=3$ & 4 & 7 & 10 \\
$T=4$ & 5 & 8 & 10 \\
\hline
\end{tabular}

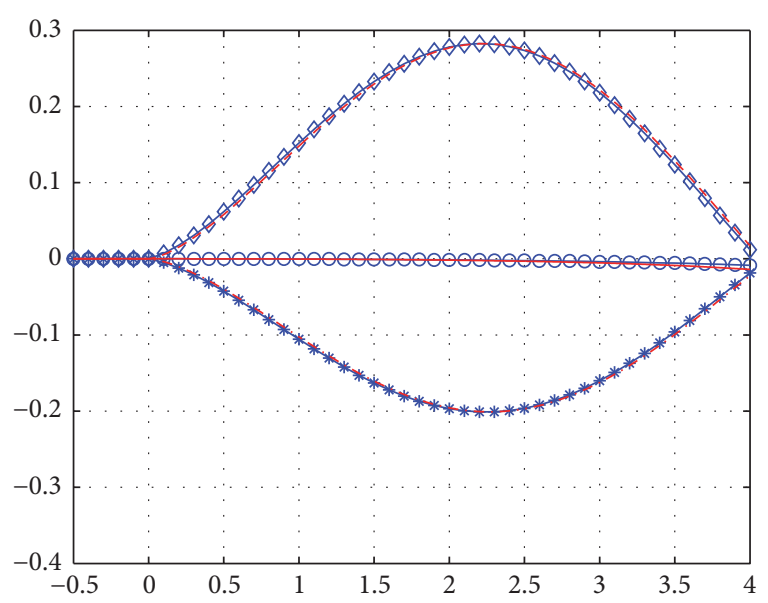

FIGURE 1: Numerical solutions by using the two methods for solving (33).

$$
\begin{aligned}
& G_{1}=0.8, \\
& G_{2}=0 .
\end{aligned}
$$

According to the iterative format (4), we take time step $h=0.1$ and use the Jacobi waveform relaxation method to solve the initial value problem (33). Then, we take time step $h=0.005$ and take the numerical solution of (33) obtained by the implicit Grünwald-Letnikov method as the approximate true solution. Then, the error of the numerical solution obtained by WR method is shown in Table 1.

The numerical solutions are plotted in Figure 1, where $*$, $\diamond$, and $\bigcirc$, respectively, are numerical solutions of $\tilde{x}_{1}(t), \tilde{x}_{2}(t)$, and $y_{1}(t)$ by using the waveform relaxation.

Table 1 and Figure 1 show that the waveform relaxation method for obtaining the solution is accurate.

Table 2 shows that the iterative allowable error is smaller, and the number of corresponding iterations is higher. If the 
iteration tolerance is the same, then the condition of iterations number is relatively stable.

Example 2. Consider the initial value problem of the linear fractional delay differential-algebraic equations

$$
\begin{aligned}
& {\left[\begin{array}{llll}
5 & 1 & 0 & 0 \\
2 & 6 & 0 & 0 \\
0 & 0 & 0 & 0 \\
0 & 0 & 0 & 0
\end{array}\right]{ }_{a}^{C} D_{0}^{\alpha} x(t)+\left[\begin{array}{cccc}
4 & 2 & 1 & 0.5 \\
1 & 5 & 0.8 & 1.2 \\
0.1 & 0.3 & 6 & 2 \\
0.2 & 0.2 & 2 & 7
\end{array}\right] x(t)} \\
& +\left[\begin{array}{llll}
3 & 1.2 & 4 & 1 \\
1.4 & 5 & 2 & 6 \\
1 & 0 & 3 & 2 \\
0 & 1 & 1 & 4
\end{array}\right] x(t-0.5)=\left[\begin{array}{c}
t \\
\sin (t) \\
1-\cos (t)
\end{array}\right], \\
& x(t)=(0,0,0,0)^{T}, \quad t \in[-0.5,0],
\end{aligned}
$$

where $\alpha=0.5, x(t)=\left(x_{1}(t), x_{2}(t), x_{3}(t), x_{4}(t)\right)^{T}$; introduce $\tilde{x}(t)=\left(x_{1}(t), x_{2}(t)\right)^{T}, y(t)=\left(x_{3}(t), x_{4}(t)\right)^{T}$; system (37) can be written as

$$
\begin{aligned}
& M_{a}^{C} D_{0}^{\alpha} \tilde{x}(t)+A \tilde{x}(t)+B y(t)+C \tilde{x}(t-0.5) \\
& \quad+D y(t-0.5)=U(t), \quad t \in[0, T], \\
& E \tilde{x}(t)+N y(t)+F \tilde{x}(t-0.5)+G y(t-0.5)=V(t), \\
& t \in[0, T],
\end{aligned}
$$

Splitting the coefficient matrix, we can construct the GaussSeidel waveform relaxation method and deduce

$$
\begin{aligned}
& M_{1}=\left[\begin{array}{ll}
5 & 0 \\
2 & 6
\end{array}\right], \\
& M_{2}=\left[\begin{array}{ll}
0 & 1 \\
0 & 0
\end{array}\right], \\
& A_{1}=\left[\begin{array}{ll}
4 & 0 \\
1 & 5
\end{array}\right], \\
& A_{2}=\left[\begin{array}{ll}
0 & 2 \\
0 & 0
\end{array}\right], \\
& B_{1}=\left[\begin{array}{ll}
1 & 0 \\
0.8 & 1.2
\end{array}\right], \\
& B_{1}=\left[\begin{array}{ll}
6 & 0 \\
2 & 7
\end{array}\right], \\
& B_{2}=\left[\begin{array}{ll}
0 & 0.5 \\
0 & 0
\end{array}\right], \\
& D_{1}=\left[\begin{array}{ll}
0 & 0 \\
0 & 0
\end{array}\right], \\
& C_{1}=\left[\begin{array}{ll}
3 & 0.3 \\
1.4 & 0.2
\end{array}\right], \\
& C_{2}=\left[\begin{array}{ll}
0 & 6 \\
0 & 1.2 \\
0 & 0
\end{array}\right],
\end{aligned}
$$

and we have

$$
t \in[-0.5,0],
$$

$$
\begin{aligned}
M & =\left[\begin{array}{ll}
5 & 1 \\
2 & 6
\end{array}\right], \\
A & =\left[\begin{array}{ll}
4 & 2 \\
1 & 5
\end{array}\right], \\
B & =\left[\begin{array}{ll}
1 & 0.5 \\
0.8 & 2
\end{array}\right], \\
C & =\left[\begin{array}{ll}
3 & 1.2 \\
1.4 & 5
\end{array}\right], \\
D & =\left[\begin{array}{ll}
4 & 1 \\
2 & 6
\end{array}\right], \\
E & =\left[\begin{array}{ll}
0.1 & 0.3 \\
0.2 & 0.2
\end{array}\right],
\end{aligned}
$$

$$
\begin{aligned}
& N=\left[\begin{array}{ll}
6 & 2 \\
2 & 7
\end{array}\right] \text {, } \\
& F=\left[\begin{array}{ll}
1 & 0 \\
0 & 1
\end{array}\right] \text {, } \\
& G=\left[\begin{array}{ll}
3 & 2 \\
1 & 4
\end{array}\right], \\
& U(t)=\left[\begin{array}{ll}
t & \sin (t)
\end{array}\right]^{T}, \\
& V(t)=1-\cos (t) \text {. }
\end{aligned}
$$


TABLE 3: The error of numerical solutions.

\begin{tabular}{lcccc}
\hline$T$ & $T=1$ & $T=2$ & $T=3$ & $T=4$ \\
\hline$\tilde{x}_{1}(t)$ & $4.1571 e-003$ & $4.9982 e-003$ & $4.7782 e-003$ & $3.4877 e-003$ \\
$\tilde{x}_{2}(t)$ & $1.2799 e-003$ & $1.2010 e-003$ & $3.2493 e-003$ & $3.0521 e-003$ \\
$y_{1}(t)$ & $4.1742 e-003$ & $4.1408 e-003$ & $1.8243 e-003$ & $1.2053 e-003$ \\
$y_{2}(t)$ & $1.0734 e-003$ & $1.2718 e-003$ & $7.4072 e-004$ & $1.1129 e-004$ \\
\hline
\end{tabular}

TABLE 4: Between the iteration allowable error and the number of iterations.

\begin{tabular}{lccc}
\hline$T$ & $1.0 \times 10^{-4}$ & $1.0 \times 10^{-6}$ & $1.0 \times 10^{-8}$ \\
\hline$T=1$ & 4 & 5 & 7 \\
$T=2$ & 3 & 5 & 7 \\
$T=3$ & 4 & 6 & 7 \\
$T=4$ & 4 & 6 & 7 \\
\hline
\end{tabular}

$$
\begin{aligned}
& N_{2}=\left[\begin{array}{ll}
0 & 2 \\
0 & 0
\end{array}\right], \\
& F_{1}=\left[\begin{array}{ll}
1 & 0 \\
0 & 1
\end{array}\right], \\
& F_{2}=\left[\begin{array}{ll}
0 & 0 \\
0 & 0
\end{array}\right], \\
& G_{1}=\left[\begin{array}{ll}
3 & 0 \\
1 & 4
\end{array}\right], \\
& G_{2}=\left[\begin{array}{ll}
0 & 2 \\
0 & 0
\end{array}\right] .
\end{aligned}
$$

According to the iterative format (4), we take time step $h=0.1$ and use the Gauss-Seidel waveform relaxation method to solve the initial value problem (37). Then, we take time step $h=0.005$ and take the numerical solution of (37) obtained by the implicit Grünwald-Letnikov method as the approximate true solution. Then, the error of the numerical solution obtained by WR method is shown in Table 3.

The numerical solutions are plotted in Figure 2, where *, $\diamond$, and $\bigcirc$, respectively, are numerical solutions of $\tilde{x}_{1}(t), \tilde{x}_{2}(t)$, and $y_{1}(t)$ by using the waveform relaxation.

Table 3 and Figure 2 show that the waveform relaxation method for obtaining the solution is accurate.

Table 4 shows that the iterative allowable error is smaller, and the number of corresponding iterations is higher. If the iteration tolerance is the same, then the condition of iterations number is relatively stable.

\section{Conclusion}

In this paper, the discrete waveform relaxation method has been extended to solve linear fractional delay differential equations. Some illustrative examples show that this method

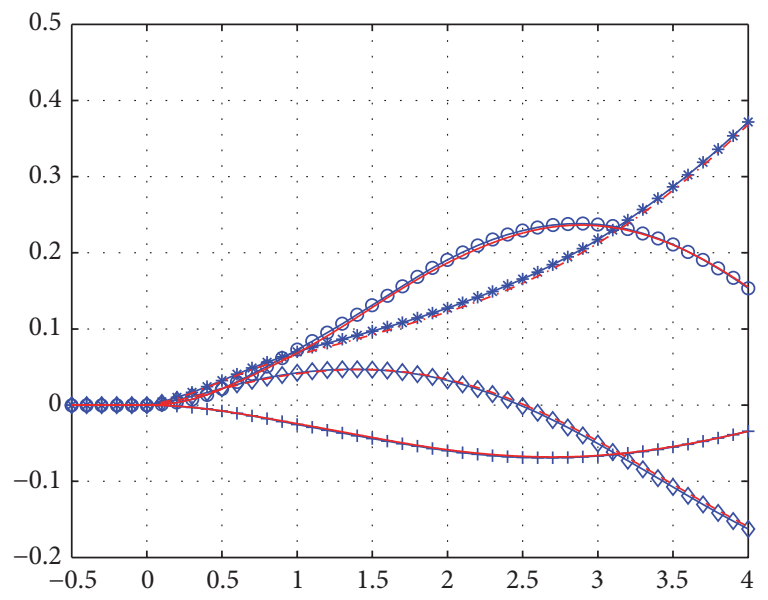

FIGURE 2: Numerical solutions by using the two methods for solving (34).

gives very good approximation to the exact solution. The discrete waveform relaxation method is a promising method to handle linear fractional delay differential-algebraic equations.

\section{Conflicts of Interest}

The authors declare that they have no conflicts of interest regarding the publication of this paper.

\section{Acknowledgments}

The authors would like to thank the Scientific Research Project of Hunan Education Department (no. 17B258) and the projects from NSF of China (nos. 11301448 and 11671343).

\section{References}

[1] X. K. Li and J. G. Peng, "Laplace transform and fractional differential equations," Applied Mathematics Letters, vol. 24, no. 24, pp. 2019-2023, 2011.

[2] S.-D. Lin and C.-H. Lu, "Laplace transform for solving some families of fractional differential equations and its applications," Advances in Difference Equations, vol. 2013, no. 1, article 137, 9 pages, 2013.

[3] L. Gaul, P. Klein, and S. Kempfle, "Impulse response function of an oscillator with fractional derivative in damping description," Mechanics Research Communications, vol. 16, no. 5, pp. 297-305, 1989.

[4] L. Gaul, P. Klein, and S. Kemple, "Damping description involving fractional operators," Mechanical Systems and Signal Processing, vol. 5, no. 2, pp. 81-88, 1991. 
[5] M. Alquran, "Analytical solutions of fractional foam drainage equation by residual power series method," Mathematical Sciences, vol. 8, no. 4, pp. 153-160, 2014.

[6] G.-C. Wu, "A fractional variational iteration method for solving fractional nonlinear differential equations," Computers and Mathematics with Applications, vol. 61, no. 8, pp. 2186-2190, 2011.

[7] H. Jafari and H. Tajadodi, "He's variational iteration method for solving fractional Riccati differential equation," International Journal of Differential Equations, vol. 2010, Article ID 764738, 8 pages, 2010.

[8] N. T. Shawagfeh, "Analytical approximate solutions for nonlinear fractional differential equations," Applied Mathematics and Computation, vol. 131, no. 2-3, pp. 517-529, 2002.

[9] M. Zurigat, S. Momani, and A. Alawneh, "Analytical approximate solutions of systems of fractional algebraic-differential equations by homotopy analysis method," Computers \& Mathematics with Applications, vol. 59, no. 3, pp. 1227-1235, 2010.

[10] R. K. Bera, P. C. Ray, A. Chakrabarti et al., "Large amplitude free vibration of a rotating non-homogeneous beam with non-linear spring and mass system," Journal of Vibration \& Acoustics, vol. 132, no. 5, pp. 35-48, 2008.

[11] C. Lubich, "Discretized fractional calculus," SIAM Journal on Mathematical Analysis, vol. 17, no. 3, pp. 704-719, 1986.

[12] K. Diethelm, N. J. Ford, and A. D. Freed, "A predictor-corrector approach for the numerical solution of fractional differential equations," Nonlinear Dynamics. An International Journal of Nonlinear Dynamics and Chaos in Engineering Systems, vol. 29, no. 1-4, pp. 3-22, 2002.

[13] C. Li, A. Chen, and J. Ye, "Numerical approaches to fractional calculus and fractional ordinary differential equation," Journal of Computational Physics, vol. 230, no. 9, pp. 3352-3368, 2011.

[14] S. Momani and K. Al-Khaled, "Numerical solutions for systems of fractional differential equations by the decomposition method," Applied Mathematics and Computation, vol. 162, no. 3, pp. 1351-1365, 2005.

[15] S. Saha Ray and R. K. Bera, "An approximate solution of a nonlinear fractional differential equation by Adomian decomposition method," Applied Mathematics and Computation, vol. 167, no. 1, pp. 561-571, 2005.

[16] S. Momani and Z. Odibat, "Numerical comparison of methods for solving linear differential equations of fractional order," Chaos, Solitons and Fractals, vol. 31, no. 5, pp. 1248-1255, 2007.

[17] P. Zhuang, F. Liu, V. Anh, and I. Turner, "Numerical methods for the variable-order fractional advection-diffusion equation with a nonlinear source term," SIAM Journal on Numerical Analysis, vol. 47, no. 3, pp. 1760-1781, 2009.

[18] F. Liu, C. Yang, and K. Burrage, "Numerical method and analytical technique of the modified anomalous subdiffusion equation with a nonlinear source term," Journal of Computational and Applied Mathematics, vol. 231, no. 1, pp. 160-176, 2009.

[19] E. Lelarasmee and A. L. Sangiovanni-Vincentelli, "The waveform relaxation method for time-domain analysis of large scale integrated circuits," IEEE Transactions on Computer-Aided Design of Integrated Circuits and Systems, vol. 1, no. 3, pp. 131$145,1982$.

[20] D. Yuan and K. Burrage, "Convergence of the parallel chaotic waveform relaxation method for stiff systems," Journal of Computational and Applied Mathematics, vol. 151, no. 1, pp. 201213, 2003.
[21] A. Lumsdaine and D. Wu, "Krylov subspace acceleration of waveform relaxation," SIAM Journal on Numerical Analysis, vol. 41, no. 1, pp. 90-111, 2003.

[22] Y.-L. Jiang and X.-L. Ding, "Waveform relaxation methods for fractional differential equations with the Caputo derivatives," Journal of Computational and Applied Mathematics, vol. 238, no. 1, pp. 51-67, 2013.

[23] X.-L. Ding and Y.-L. Jiang, "A windowing waveform relaxation method for time-fractional differential equations," Communications in Nonlinear Science and Numerical Simulation, vol. 30, no. 1-3, pp. 139-150, 2016.

[24] W.-S. Wang and S.-F. Li, "Convergence of waveform relaxation methods for neutral delay differential equations," Mathematical and Computer Modelling, vol. 48, no. 11-12, pp. 1875-1887, 2008.

[25] B. Zubik-Kowal and S. Vandewalle, "Waveform relaxation for functional-differential equations," SIAM Journal on Scientific Computing, vol. 21, no. 1, pp. 207-226, 1999.

[26] Y.-L. Jiang, "On time-domain simulation of lossless transmission lines with nonlinear terminations," SIAM Journal on Numerical Analysis, vol. 42, no. 3, pp. 1018-1031, 2004.

[27] Y.-L. Jiang and O. Wing, "A note on the spectra and pseudospectra of waveform relaxation operators for linear differentialalgebraic equations," SIAM Journal on Numerical Analysis, vol. 38, no. 1, pp. 186-201, 2000.

[28] W. Bao and Y. Z. Song, "Two stage waveform relaxation method for the initial value problems of differential-algebraic equations," Journal of Computational and Applied Mathematics, vol. 236, no. 6, pp. 1123-1136, 2011.

[29] R. Jeltsch and B. Pohl, "Waveform relaxation with overlapping splittings," SIAM Journal on Scientific Computing, vol. 16, no. 1, pp. 40-49, 1995.

[30] B. Pohl, "On the convergence of the discretized multi-splitting waveform relaxation algorithm," Applied Numerical Mathematics. An IMACS Journal, vol. 11, no. 1-3, pp. 251-258, 1993.

[31] R. H. Chan, M. K. Ng, and X.-Q. Jin, "Strang-type preconditioners for systems of LMF-based ODE codes," IMA Journal of Numerical Analysis, vol. 21, no. 2, pp. 451-462, 2001.

[32] H. Zhang, "A note on windowing for the waveform relaxation method," Applied Mathematics and Computation, vol. 76, no. 1, pp. 49-63, 1996.

[33] B. Ibiş and M. Bayram, "Numerical comparison of methods for solving fractional differential-algebraic equations (FDAEs)," Computers and Mathematics with Applications, vol. 62, no. 8, pp. 3270-3278, 2011.

[34] B. İbiș, M. Bayram, and A. G. Ağargün, "Applications of fractional differential transform method to fractional differentialalgebraic equations," European Journal of Pure \& Applied Mathematics, vol. 4, no. 2, pp. 129-141, 2011.

[35] X.-L. Ding and Y.-L. Jiang, "Waveform relaxation method for fractional differential-algebraic equations," Fractional Calculus and Applied Analysis, vol. 17, no. 3, pp. 585-604, 2014.

[36] Y. Fu, H. Liu, and A. Xiao, "Convergence of variational iteration method for fractional delay integrodifferential-algebraic equations," Mathematical Problems in Engineering, vol. 2017, Article ID 6749643, 10 pages, 2017. 


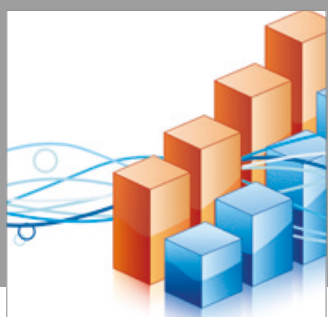

Advances in

Operations Research

vatersals

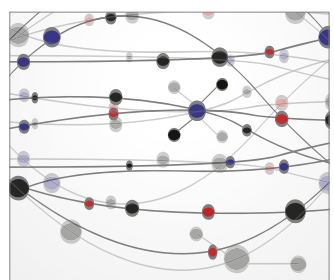

\section{The Scientific} World Journal
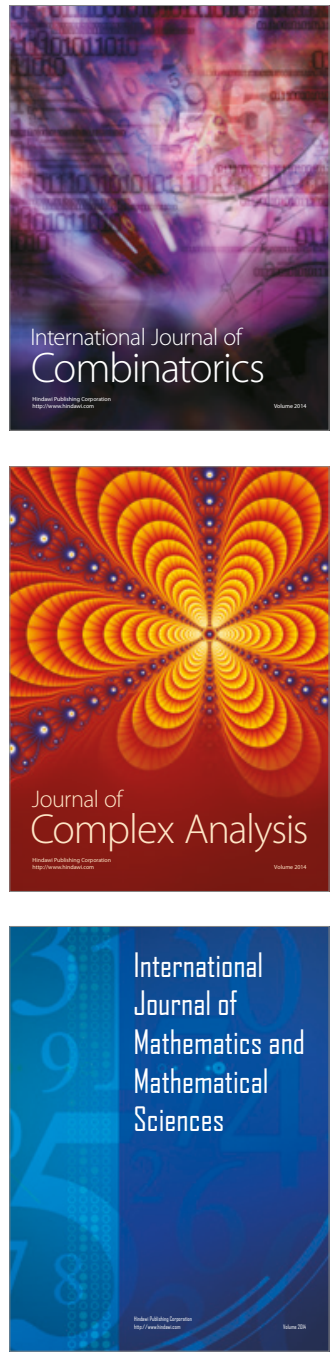
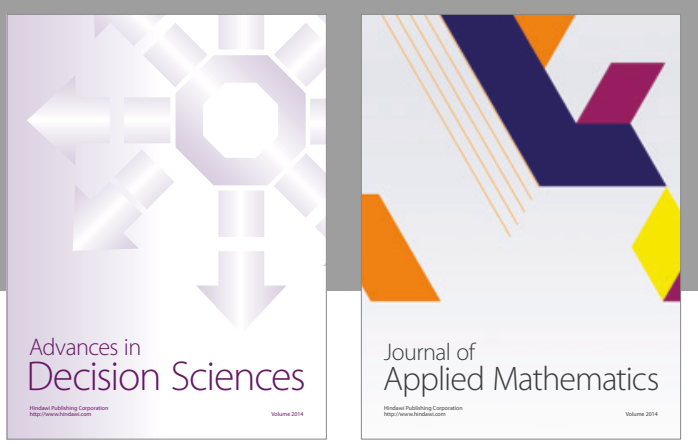

Algebra

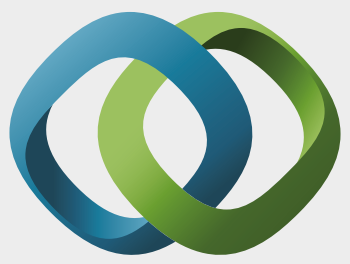

\section{Hindawi}

Submit your manuscripts at

https://www.hindawi.com
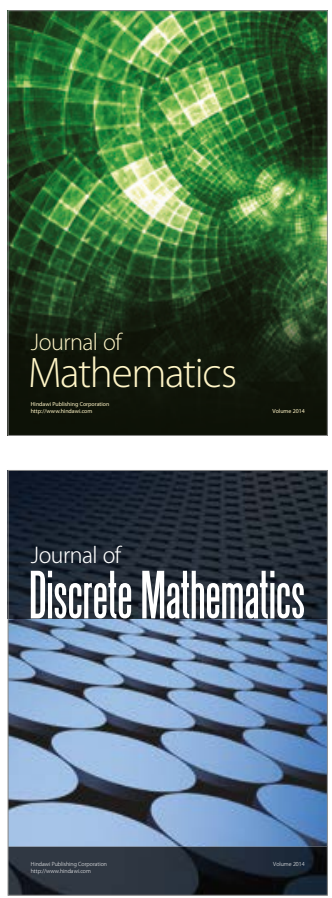

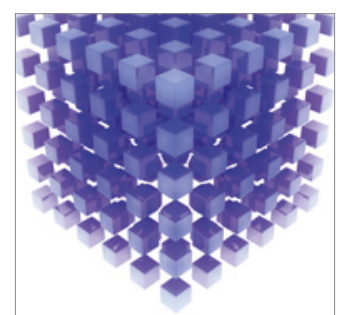

Mathematical Problems in Engineering
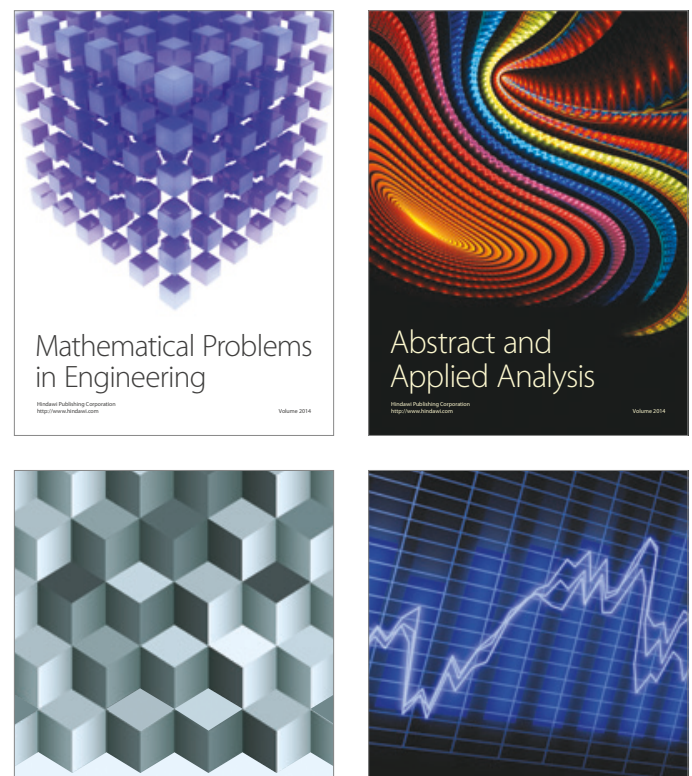

Journal of

Function Spaces

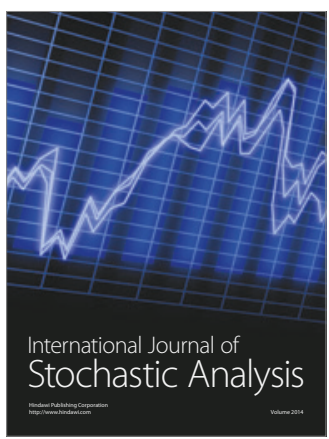

Probability and Statistics
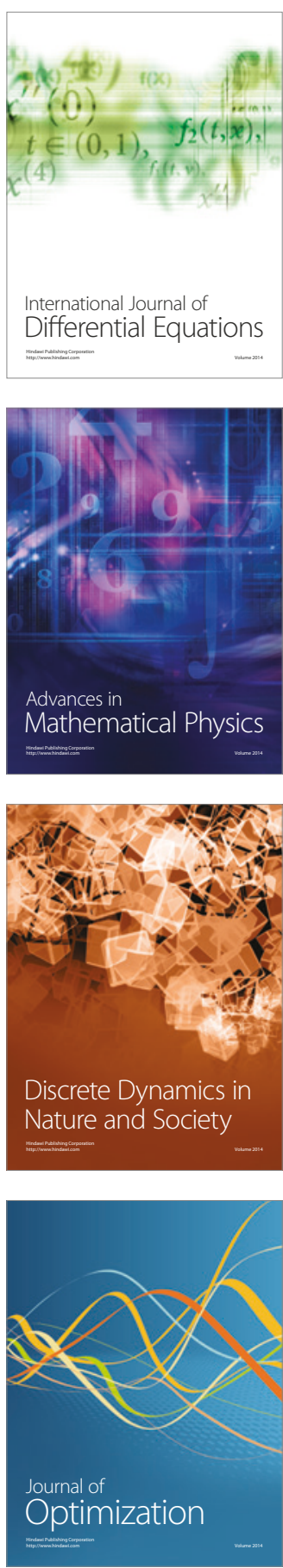